\title{
Minimization of Vectors of Curvilinear Functionals on Second-Order Jet Bundle: Dual Program Theory
}

\author{
Mihai Postolache \\ Faculty of Applied Sciences, University "Politehnica" of Bucharest, Splaiul Independentei 313, \\ 060042 Bucharest, Romania \\ Correspondence should be addressed to Mihai Postolache, emscolar@yahoo.com
}

Received 6 December 2011; Accepted 27 December 2011

Academic Editor: Ngai-Ching Wong

Copyright (C) 2012 Mihai Postolache. This is an open access article distributed under the Creative Commons Attribution License, which permits unrestricted use, distribution, and reproduction in any medium, provided the original work is properly cited.

\begin{abstract}
A previous paper (2011), Pitea and Postolache, considered the problem of minimization of vectors of curvilinear functionals (well known as mechanical work), thought as multitime multiobjective variational problem, subject to PDE and/or PDI constraints. They have chosen the suitable framework offered by the second-order jet bundle, and initiated an optimization theory for this class of problems by introducing necessary conditions. As natural continuation of these results, the present work introduces a dual program theory, the general setting, and the theory which is new as a whole, containing our results.
\end{abstract}

\section{Introduction}

Fractional programming problems arise in wide areas of research in pure and applied sciences and in the new technology as well. We have in mind portfolio selection, stock cutting, game theory, and various decision problems in management science. The study of duality for this class of problems is an active area of research due to its wide scope of applications, see [1] by Chinchuluun and Pardalos, for an excellent paper in multiobjective optimization.

The duality theory for multiobjective optimization problems based on a vector-valued Lagrangian function were extensively investigated in [2] by Bitran, and [3] by Tanino and Sawaragi. Several authors were interested in this problem, see [4] by Egudo, [5] by Mond and Husain, [6] by Weir and Mond. Later, an interesting and rich duality theory for such problems was developed by Aghezzaf and Hachimi, [7], Preda [8, 9], and recently, duality problems were studied in [10] by Boț and Grad, [1] by Chinchuluun and Pardalos, [11] by Pitea et al.

In the problems of our study, the objective function is of curvilinear integral type and could mould the mechanical work when we have in mind design problems in engineering. 
Also, such kind of problems proved to be useful tools for minimizing the cost or maximizing the profit when we discuss economical problems.

Motivated by the study reported above, in a very recent work [12], Pitea and Postolache considered the problem of minimization of vectors of curvilinear functionals (well known as mechanical work), thought as multitime multiobjective variational problem, subject to PDE and/or PDI constraints. They have chosen the suitable framework offered by the second-order jet bundle and initiated an optimization theory for this class of problems by introducing necessary conditions. As natural continuation of these results, the present work introduces a dual program theory. It is organized as follows. Next, in Section 2, we introduce the general setting, while in Section 3 we prove our results. Finally, we conclude the paper.

\section{General Setting}

Let $(T, h)$ and $(M, g)$ be Riemannian manifolds of dimensions $p$ and $n$, respectively. The local coordinates on $T$ and $M$ will be written $t=\left(t^{\alpha}\right)$ and $x=\left(x^{i}\right)$, respectively. Let $J^{2}(T, M)$ be the second-order jet bundle associated to $T$ and $M$, see [13].

Throughout this work, we use the customary relations between two vectors of the same dimension, [11]. With the product-order relation on $\mathbb{R}^{p}$, the hyperparallelepiped $\Omega_{t_{0}, t_{1}}$, in $\mathbb{R}^{p}$, with the diagonal opposite points $t_{0}=\left(t_{0}^{1}, \ldots, t_{0}^{p}\right)$ and $t_{1}=\left(t_{1}^{1}, \ldots, t_{1}^{p}\right)$, can be written as interval $\left[t_{0}, t_{1}\right]$. Suppose $\gamma_{t_{0}, t_{1}}$ is a piecewise $C^{2}$-class curve joining the points $t_{0}$ and $t_{1}$.

To simplify the notations, denote by

$$
x_{\gamma}(t)=\frac{\partial x}{\partial t^{\gamma}}(t), \quad \gamma=\overline{1, p}, \quad x_{\theta \sigma}(t)=\frac{\partial^{2} x}{\partial t^{\theta} \partial t^{\sigma}}(t), \quad \theta, \sigma=\overline{1, p}
$$

the partial velocities and partial accelerations, respectively. Also, in our subsequent theory, we will set $\pi_{x}(t)=\left(t, x(t), x_{\gamma}(t), x_{\theta \sigma}(t)\right)$.

On $J^{2}(T, M)$ with values on $\mathbb{R}^{r}$, consider the closed Lagrange 1 -forms densities of $C^{\infty}$ class:

$$
f_{\alpha}=\left(f_{\alpha}^{\ell}\right), \quad k_{\alpha}=\left(k_{\alpha}^{\ell}\right), \quad \ell=\overline{1, r}, \alpha=\overline{1, p}
$$

which determine the following path-independent functionals:

$$
F^{\ell}(x(\cdot))=\int_{\gamma_{t_{0}, t_{1}}} f_{\alpha}^{\ell}\left(\pi_{x}(t)\right) d t^{\alpha}, \quad K^{\ell}(x(\cdot))=\int_{\gamma_{t_{0}, t_{1}}} k_{\alpha}^{\ell}\left(\pi_{x}(t)\right) d t^{\alpha} .
$$

The closeness conditions (complete integrability conditions) are

$$
D_{\beta} f_{\alpha}^{\ell}=D_{\alpha} f_{\beta^{\prime}}^{\ell} \quad D_{\beta} k_{\alpha}^{\ell}=D_{\alpha} k_{\beta^{\prime}}^{\ell} \quad \alpha, \beta=\overline{1, p}, \alpha \neq \beta, \ell=\overline{1, r},
$$

where $D_{\beta}$ is the total derivative.

We accept that the Lagrange matrix densities:

$$
g=\left(g_{a}^{b}\right): J^{2}(T, M) \longrightarrow \mathbb{R}^{m d}, \quad a=\overline{1, d}, b=\overline{1, m}, m<n,
$$


of $C^{\infty}$-class define the partial differential inequations (PDI) of evolution

$$
g\left(\pi_{x}(t)\right) \leqq 0, \quad t \in \Omega_{t_{0}, t_{1}}
$$

and the Lagrange matrix densities,

$$
h=\left(h_{a}^{b}\right): J^{2}(T, M) \longrightarrow \mathbb{R}^{q d}, \quad a=\overline{1, s}, b=\overline{1, q}, q<n,
$$

define the partial differential equations (PDE), of evolution

$$
h\left(\pi_{x}(t)\right)=0, \quad t \in \Omega_{t_{0}, t_{1}} .
$$

For each $\ell=\overline{1, r}$, suppose $K^{\ell}(x(\cdot))>0$, and consider

$$
\frac{F(x(\cdot))}{K(x(\cdot))}=\left(\frac{F^{1}(x(\cdot))}{K^{1}(x(\cdot))}, \ldots, \frac{F^{r}(x(\cdot))}{K^{r}(x(\cdot))}\right) .
$$

With conditions (2.6) and (2.8), we denote by

$$
\mathcal{F}\left(\Omega_{t_{0}, t_{1}}\right)=\left\{x \in C^{\infty}\left(\Omega_{t_{0}, t_{1}}, M\right) \mid x\left(t_{0}\right)=x_{0}, x\left(t_{1}\right)=x_{1}, g\left(\pi_{x}(t)\right) \leqq 0, h\left(\pi_{x}(t)\right)=0, t \in \Omega_{t_{0}, t_{1}}\right\}
$$

the set of all feasible solutions of problem:

$$
(\mathrm{MFP})\left\{\begin{array}{l}
\min \frac{F(x(\cdot))}{K(x(\cdot))} \\
\text { subject to } x(\cdot) \in \mathcal{F}\left(\Omega_{t_{0}, t_{1}}\right),
\end{array}\right.
$$

a PDI- and/or PDE-constrained minimum problem.

In [12], we introduced necessary efficiency conditions for problem (MFP). The aim of this work is to introduce and study two dual programs:

(1) the multitime multiobjective fractional variational problem (MFP) of minimizing a vector of quotients of path-independent curvilinear functionals; 
(2) the multiobjective variational dual problem:

$$
(\operatorname{MFD})\left\{\begin{array}{l}
\max _{y(\cdot)}\left(\frac{F(y(\cdot))}{K(y(\cdot))}\right) \\
\text { subject to } \\
\quad \Lambda_{\ell}^{10} \frac{\partial f_{\alpha}^{\ell}}{\partial y}\left(\pi_{y}(t)\right)-\Lambda_{\ell}^{20} \frac{\partial k_{\alpha}^{\ell}}{\partial y}\left(\pi_{y}(t)\right)+\left\langle\mu_{\alpha}(t), \frac{\partial g}{\partial y}\left(\pi_{y}(t)\right)\right\rangle \\
+\left\langle v_{\alpha}(t), \frac{\partial h}{\partial y}\left(\pi_{y}(t)\right)\right\rangle-D_{\gamma}\left(\Lambda_{\ell}^{10} \frac{\partial f_{\alpha}^{\ell}}{\partial y_{\gamma}}\left(\pi_{y}(t)\right)-\Lambda_{\ell}^{20} \frac{\partial k_{\alpha}^{\ell}}{\partial y_{\gamma}}\left(\pi_{y}(t)\right)\right. \\
\left.\quad+\left\langle\mu_{\alpha}(t), \frac{\partial g}{\partial y_{\gamma}}\left(\pi_{y}(t)\right)\right\rangle+\left\langle v_{\alpha}(t), \frac{\partial h}{\partial y_{\gamma}}\left(\pi_{y}(t)\right)\right\rangle\right) \\
\quad+D_{\theta \sigma}^{2}\left(\left\langle\lambda, \frac{\partial f_{\alpha}}{\partial x_{\theta \sigma}}\left(\pi_{y}(t)\right)\right\rangle+\left\langle\mu_{\alpha}(t), \frac{\partial g}{\partial x_{\theta \sigma}}\left(\pi_{y}(t)\right)\right\rangle\right. \\
\left.\quad+\left\langle v_{\alpha}(t), \frac{\partial h}{\partial x_{\theta \sigma}}\left(\pi_{y}(t)\right)\right\rangle\right)=0, \quad t \in \Omega_{t_{0}, t_{1}}, \alpha=\overline{1, p} \\
\left\langle\mu_{\alpha}(t), g\left(\pi_{y}(t)\right)\right\rangle+\left\langle v_{\alpha}(t), h\left(\pi_{y}(t)\right)\right\rangle \geqq 0, \quad \alpha=\overline{1, p}, t \in \Omega_{t_{0}, t_{1}} \\
\Lambda^{10} \geq 0, \quad
\end{array}\right.
$$

taking into account that the functions $x(t)$ and $y(t)$ have to satisfy the boundary conditions $x\left(t_{0}\right)=x_{0}, x\left(t_{1}\right)=x_{1}$, or $\left.x(t)\right|_{\partial \Omega_{t_{0}, t_{1}}}=x=$ given, respectively, $y\left(t_{0}\right)=x_{0}$, $y\left(t_{1}\right)=x_{1}$, or $\left.y(t)\right|_{\partial \Omega_{t_{0}, t_{1}}}=x=$ given, the partial differential inequations of evolution (2.6), and the partial differential equations of evolution (2.8).

To develop the theory in our main section, we need the notion of efficient solution.

Definition 2.1. A feasible solution $x^{\circ}(\cdot) \in \mathcal{F}\left(\Omega_{t_{0}, t_{1}}\right)$ is called efficient solution for the program (MFP) if and only if for any feasible solution $x(\cdot) \in \mathcal{F}\left(\Omega_{t_{0}, t_{1}}\right)$, one has the implication:

$$
\frac{F(x(\cdot))}{K(x(\cdot))} \geqq \frac{F\left(x^{\circ}(\cdot)\right)}{K\left(x^{\circ}(\cdot)\right)} \Longrightarrow \frac{F(x(\cdot))}{K(x(\cdot))}=\frac{F\left(x^{\circ}(\cdot)\right)}{K\left(x^{\circ}(\cdot)\right)}
$$

\section{Main Results}

To state our results, we have to introduce an appropriate generalized convexity. For this purpose, consider $\rho$ be a real number, $b: C^{\infty}\left(\Omega_{t_{0}, t_{1}}, M\right) \times C^{\infty}\left(\Omega_{t_{0}, t_{1}}, M\right) \rightarrow[0, \infty)$ a functional, and $a=\left(a_{\alpha}\right), \alpha=\overline{1, p}$, a closed 1-form. To $a$ we associate the curvilinear integral

$$
A(x(\cdot))=\int_{\gamma_{t_{0}, t_{1}}} a_{\alpha}\left(\pi_{x}(t)\right) d t^{\alpha}
$$


Definition 3.1. The functional $A$ is called [strictly] $(\rho, b)$-quasiinvex at the point $x^{\circ}(\cdot)$ if there exists a vector function $\eta: J^{2}\left(\Omega_{t_{0}, t_{1}}, M\right) \times J^{2}\left(\Omega_{t_{0}, t_{1}}, M\right) \rightarrow \mathbb{R}^{n}$, vanishing at the point $\left(\pi_{x^{\circ}}(t), \pi_{x^{\circ}}(t)\right)$, and the function $\theta$ defined on $C^{\infty}\left(\Omega_{t_{0}, t_{1}}, M\right) \times C^{\infty}\left(\Omega_{t_{0}, t_{1}}, M\right)$ to $\mathbb{R}^{n}$, such that for any $x(\cdot)\left[x(\cdot) \neq x^{\circ}(\cdot)\right]$, the following implication holds:

$$
\begin{aligned}
&\left(A(x(\cdot)) \leq A\left(x^{\circ}(\cdot)\right)\right) \\
& \Longrightarrow\left(b x\left((\cdot), x^{\circ}(\cdot)\right) \int_{\gamma_{t_{0}, t_{1}}}\right.\left\langle\left\langle\eta\left(\pi_{x}(t), \pi_{x^{\circ}}(t)\right), \frac{\partial a_{\alpha}}{\partial x}\left(\pi_{x^{\circ}}(t)\right)\right\rangle\right. \\
&+\left\langle D_{\gamma} \eta\left(\pi_{x}(t), \pi_{x^{\circ}}(t)\right), \frac{\partial a_{\alpha}}{\partial x_{\gamma}}\left(\pi_{x^{\circ}}(t)\right)\right\rangle \\
&+\left.\left\langle D_{\theta \sigma}^{2} \eta\left(\pi_{x}(t), \pi_{x^{\circ}}(t)\right), \frac{\partial a_{\alpha}}{\partial x_{\theta \sigma}}\left(\pi_{x^{\circ}}(t)\right)\right\rangle\right] d t^{\alpha} \\
& {\left.[<] \leq-\rho b\left(x(\cdot), x^{\circ}(\cdot)\right)\left\|\theta\left(x(\cdot), x^{\circ}(\cdot)\right)\right\|^{2}\right) . }
\end{aligned}
$$

The notion of quasiinvexity is used, in appropriate forms, in recent works for studies of some multiobjective programming problems, for example, see [14] by Nahak and Mohapatra.

Let us denote by $\varpi\left(x^{\circ}(\cdot)\right)$ the minimizing functional vector of problem (MFP) at the point $x^{\circ}(\cdot) \in \mathcal{F}\left(\Omega_{t_{0}, t_{1}}\right)$ and by $\left(\delta\left(y(\cdot), y_{\gamma}(\cdot), \Lambda^{10}, \Lambda^{20}, \mu(\cdot), v(\cdot)\right)\right.$ the maximizing functional vector of dual (MFD) at the point $\left(y(\cdot), y_{\gamma}(\cdot), \Lambda^{10}, \Lambda^{20}, \mu(\cdot), v(\cdot)\right) \in \Delta$, where $\Delta$ is the domain of problem (MFD).

Theorem 3.2 (Weak Duality). Let $x^{\circ}(\cdot)$ be a feasible solution of the problem (MFP) and let $y(\cdot)$ be an efficient solution of problem (MFP). Assume that the following conditions are fulfilled:

(a) $\Lambda_{\ell}^{10}>0, \Lambda_{\ell}^{20}>0, \Lambda_{\ell}^{10} F^{\ell}(y(\cdot))-\Lambda_{\ell}^{20} K^{\ell}(y(\cdot))=0, \ell=\overline{1, r}$;

(b) for any $\ell=\overline{1, r}$, the functional $F^{\ell}(x(\cdot))$ is $\left(\rho^{\prime \ell}, b\right)$-quasiinvex at the point $y(\cdot)$ and the functional $-K^{\ell}(x(\cdot))$ is $\left(\rho^{\prime \prime \ell}, b\right)$-quasiinvex at the point $y(\cdot)$ with respect to $\eta$ and $\theta$;

(c) the functional,

$$
\int_{\gamma_{t_{0}, t_{1}}}\left[\left\langle\mu_{\alpha}(t), g\left(\pi_{x}(t)\right)\right\rangle+\left\langle v_{\alpha}(t), h\left(\pi_{x}(t)\right)\right\rangle\right] d t^{\alpha},
$$

is $\left(\rho^{\prime \prime \prime}, b\right)$-quasiinvex at the point $y(\cdot)$ with respect to $\eta$ and $\theta$;

(d) either the functional of (b) or the functional of (c) is strictly quasiinvex;

(e) $\rho^{\prime \ell} \Lambda_{\ell}^{10}+\rho^{\prime \prime \ell} \Lambda_{\ell}^{20}+\rho^{\prime \prime \prime} \geq 0$.

Then, the inequality $\varpi\left(x^{\circ}(\cdot)\right) \leq \delta\left(y(\cdot), y_{\gamma}(\cdot), \Lambda^{10}, \Lambda^{20}, \mu(\cdot), v(\cdot)\right)$ is false. 
Proof. From condition (b), it follows that

$$
\begin{aligned}
& \left(F^{\ell}\left(x^{\circ}(\cdot)\right) F^{\ell}(y(\cdot))\right) \\
& \Longrightarrow\left(b ( x ^ { \circ } ( \cdot ) , y ( \cdot ) ) \int _ { \gamma _ { t _ { 0 } , t _ { 1 } } } \left[\left\langle\eta\left(\pi_{x^{\circ}}(t), \pi_{y}(t)\right), \frac{\partial f_{\alpha}^{\ell}}{\partial y}\left(\pi_{y}(t)\right)\right\rangle\right.\right. \\
& +\left\langle D_{\gamma} \eta\left(\pi_{x^{\circ}}(t), \pi_{y}(t)\right), \frac{\partial f_{\alpha}^{\ell}}{\partial y_{\gamma}}\left(\pi_{y}(t)\right)\right\rangle \\
& \left.+\left\langle D_{\theta \sigma}^{2} \eta\left(\pi_{x^{\circ}}(t), \pi_{y}(t)\right), \frac{\partial f_{\alpha}^{\ell}}{\partial y_{\theta \sigma}}\left(\pi_{y}(t)\right)\right\rangle\right] d t^{\alpha} \\
& \left.\leqq-\rho^{\prime \ell} b\left(x^{\circ}(\cdot), y(\cdot)\right)\left\|\theta\left(x^{\circ}(\cdot), y(\cdot)\right)\right\|^{2}\right), \quad \ell=\overline{1, r} \\
& \left(-K^{\ell}\left(x^{\circ}(\cdot)\right)-K^{\ell}(y(\cdot))\right) \\
& \Longrightarrow\left(b ( x ^ { \circ } ( \cdot ) , y ( \cdot ) ) \int _ { \gamma _ { t _ { 0 } , t _ { 1 } } } \left[\left\langle-\eta\left(\pi_{x^{\circ}}(t), \pi_{y}(t)\right), \frac{\partial k_{\alpha}^{\ell}}{\partial y}\left(\pi_{y}(t)\right)\right\rangle\right.\right. \\
& -\left\langle D_{\gamma} \eta\left(\pi_{x^{\circ}}(t), \pi_{y}(t)\right), \frac{\partial k_{\alpha}^{\ell}}{\partial y_{\gamma}}\left(\pi_{y}(t)\right)\right\rangle \\
& \left.-\left\langle D_{\theta \sigma}^{2} \eta\left(\pi_{x^{\circ}}(t), \pi_{y}(t)\right), \frac{\partial k_{\alpha}^{\ell}}{\partial y_{\theta \sigma}}\left(\pi_{y}(t)\right)\right\rangle\right] d t^{\alpha} \\
& \left.\leqq \rho^{\prime \prime \ell} b\left(x^{\circ}(\cdot), y(\cdot)\right)\left\|\theta\left(x^{\circ}(\cdot), y(\cdot)\right)\right\|^{2}\right), \quad \ell=\overline{1, r}
\end{aligned}
$$

We multiply (3.4) by $\Lambda_{\ell}^{10}>0$ and (3.5) by $\Lambda_{\ell}^{20}>0$. We make the sum and we obtain the following implications:

$$
\begin{aligned}
&\left(\Lambda_{\ell}^{10} F^{\ell}\left(x^{\circ}(\cdot)\right)-\Lambda_{\ell}^{20} K^{\ell}\left(x^{\circ}(\cdot)\right) \leqq 0\right) \\
& \Longrightarrow\left(b\left(x^{\circ}(\cdot), y(\cdot)\right) \int_{\gamma_{t_{0} t_{1}}}\{\right.\left\langle\eta\left(\pi_{x^{\circ}}(t), \pi_{y}(t)\right), \Lambda_{\ell}^{10} \frac{\partial f_{\alpha}^{\ell}}{\partial y}\left(\pi_{y}(t)\right)-\Lambda_{\ell}^{20} \frac{\partial k_{\alpha}^{\ell}}{\partial y}\left(\pi_{y}(t)\right)\right\rangle \\
&+\left\langle D_{\gamma} \eta\left(\pi_{x^{\circ}}(t), \pi_{y}(t)\right), \Lambda_{\ell}^{10} \frac{\partial f_{\alpha}^{\ell}}{\partial y_{\gamma}}\left(\pi_{y}(t)\right)-\Lambda_{\ell}^{20} \frac{\partial k_{\alpha}^{\ell}}{\partial y_{\gamma}}\left(\pi_{y}(t)\right)\right\rangle \\
&+\left.\left\langle D_{\theta \sigma}^{2} \eta\left(\pi_{x^{\circ}}(t), \pi_{y}(t)\right), \Lambda_{\ell}^{10} \frac{\partial f_{\alpha}^{\ell}}{\partial y_{\theta \sigma}}\left(\pi_{y}(t)\right)-\Lambda_{\ell}^{20} \frac{\partial k_{\alpha}^{\ell}}{\partial y_{\theta \sigma}}\left(\pi_{y}(t)\right)\right\rangle\right\} d t^{\alpha} \\
&\left.\leqq-b\left(x^{\circ}(\cdot), y(\cdot)\right)\left\|\theta\left(x^{\circ}(\cdot), y(\cdot)\right)\right\|^{2}\left(\rho^{\prime \ell} \Lambda_{\ell}^{10}+\rho^{\prime \ell} \Lambda_{\ell}^{20}\right)\right)
\end{aligned}
$$


According to hypothesis (c), we have

$$
\begin{gathered}
\left(\int_{\gamma_{t_{0}, t_{1}}}\left[\left\langle\mu_{\alpha}(t), g\left(\pi_{x^{\circ}}(t)\right)\right\rangle+\left\langle v_{\alpha}(t), h\left(\pi_{x^{\circ}}(t)\right)\right\rangle\right] d t^{\alpha}\right. \\
\left.\leqq \int_{\gamma_{t_{0}, t_{1}}}\left[\left\langle\mu_{\alpha}(t), g\left(\pi_{y}(t)\right)\right\rangle+\left\langle v_{\alpha}(t), h\left(\pi_{y}(t)\right)\right\rangle\right] d t^{\alpha}\right) \\
\Longrightarrow\left(b ( x ^ { \circ } ( \cdot ) , y ( \cdot ) ) \int _ { \gamma _ { t _ { 0 } , t _ { 1 } } } \left[\left\langle\eta\left(\pi_{x^{\circ}}(t), \pi_{y}(t)\right),\left\langle\mu_{\alpha}(t), \frac{\partial g}{\partial y}\left(\pi_{y}(t)\right)\right\rangle\right.\right.\right. \\
\left.+\left\langle v_{\alpha}(t), \frac{\partial h}{\partial y}\left(\pi_{y}(t)\right)\right\rangle\right\rangle+\left\langle D_{\gamma} \eta\left(\pi_{x^{\circ}}(t), \pi_{y}(t)\right)\right\rangle, \\
\left.\left\langle\mu_{\alpha}(t), \frac{\partial g}{\partial y_{\gamma}}\left(\pi_{y}(t)\right)\right\rangle+\left\langle v_{\alpha}(t), \frac{\partial h}{\partial y_{\gamma}}\left(\pi_{y}(t)\right)\right\rangle\right\rangle \\
+\left\langle D_{\theta \sigma}^{2} \eta\left(\pi_{x^{\circ}}(t), \pi_{y}(t)\right)\right\rangle,\left\langle\mu_{\alpha}(t), \frac{\partial g}{\partial y_{\theta \sigma}}\left(\pi_{y}(t)\right)\right\rangle \\
\left.\left.+\left\langle v_{\alpha}(t), \frac{\partial h}{\partial y_{\theta \sigma}}\left(\pi_{y}(t)\right)\right\rangle\right\rangle\right] d t^{\alpha} \\
\left.\leqq-\rho^{\prime \prime \prime} b\left(x^{\circ}(\cdot), y(\cdot)\right)\left\|\theta\left(x^{\circ}(\cdot), y(\cdot)\right)\right\|^{2}\right) .
\end{gathered}
$$

Making the sum of the implications (3.6) and (3.7), it follows that

$$
\begin{aligned}
& \left(\Lambda_{\ell}^{10} F^{\ell}\left(x^{\circ}(\cdot)\right)-\Lambda_{\ell}^{20} K^{\ell}\left(x^{\circ}(\cdot)\right)\right. \\
& +\int_{\gamma_{t_{0}, t_{1}}}\left[\left\langle\mu_{\alpha}(t), g\left(\pi_{x^{\circ}}(t)\right)\right\rangle+\left\langle v_{\alpha}(t), h\left(\pi_{x^{\circ}}(t)\right)\right\rangle\right] d t^{\alpha} \\
& \left.-\int_{\gamma_{t_{0}, t_{1}}}\left[\left\langle\mu_{\alpha}(t), g\left(\pi_{y}(t)\right)\right\rangle+\left\langle v_{\alpha}(t), h\left(\pi_{y}(t)\right)\right\rangle\right] d t^{\alpha} \leqq 0\right) \\
& \Longrightarrow\left(b ( x ^ { \circ } ( \cdot ) , y ( \cdot ) ) \int _ { \gamma _ { t _ { 0 } , t _ { 1 } } } \left[\left\langle\eta\left(\pi_{x^{\circ}}(t), \pi_{y}(t)\right), \Lambda_{\ell}^{10} \frac{\partial f_{\alpha}^{\ell}}{\partial y}\left(\pi_{y}(t)\right)-\Lambda_{\ell}^{20} \frac{\partial k_{\alpha}^{\ell}}{\partial y}\left(\pi_{y}(t)\right)\right.\right.\right. \\
& \left.+\left\langle\mu_{\alpha}(t), \frac{\partial g}{\partial y}\left(\pi_{y}(t)\right)\right\rangle+\left\langle v_{\alpha}(t), \frac{\partial h}{\partial y}\left(\pi_{y}(t)\right)\right\rangle\right\rangle \\
& +\left\langle D_{\gamma} \eta\left(\pi_{x^{\circ}}(t), \pi_{y}(t)\right), \Lambda_{\ell}^{10} \frac{\partial f_{\alpha}^{\ell}}{\partial y_{\gamma}}\left(\pi_{y}(t)\right)-\Lambda_{\ell}^{20} \frac{\partial k_{\alpha}^{\ell}}{\partial y_{\gamma}}\left(\pi_{y}(t)\right)\right.
\end{aligned}
$$




$$
\begin{aligned}
&+\left.\left\langle\mu_{\alpha}(t), \frac{\partial g}{\partial y_{\gamma}}\left(\pi_{y}(t)\right)\right\rangle+\left\langle v_{\alpha}(t), \frac{\partial h}{\partial y_{\gamma}}\left(\pi_{y}(t)\right)\right\rangle\right\rangle \\
&+\left\langle D_{\theta \sigma}^{2} \eta\left(\pi_{x^{\circ}}(t), \pi_{y}(t)\right), \quad \Lambda_{\ell}^{10} \frac{\partial f_{\alpha}^{\ell}}{\partial y_{\theta \sigma}}\left(\pi_{y}(t)\right)-\Lambda_{\ell}^{20} \frac{\partial k_{\alpha}^{\ell}}{\partial y_{\theta \sigma}}\left(\pi_{y}(t)\right)\right. \\
&+\left.\left.\left\langle\mu_{\alpha}(t), \frac{\partial g}{\partial y_{\theta \sigma}}\left(\pi_{y}(t)\right)\right\rangle+\left\langle v_{\alpha}(t), \frac{\partial h}{\partial y_{\theta \sigma}}\left(\pi_{y}(t)\right)\right\rangle\right\rangle\right] d t^{\alpha} \\
&\left.<-b\left(x^{\circ}(\cdot), y(\cdot)\right)\left\|\theta\left(x^{\circ}(\cdot), y(\cdot)\right)\right\|^{2}\left(\rho^{\prime \ell} \Lambda_{\ell}^{10}+\rho^{\prime \prime \ell} \Lambda_{\ell}^{20}+\rho^{\prime \prime \prime}\right)\right) .
\end{aligned}
$$

Since $b\left(x^{\circ}(\cdot), y(\cdot)\right)>0$, we obtain

$$
\begin{aligned}
\int_{\gamma_{t_{0}, t_{1}}}\left[\left\langle\eta \pi_{x^{\circ}}(t), \pi_{y}(t), \frac{\partial V_{\alpha}}{\partial y \pi_{y}}(t), \lambda, \mu(t), v(t)\right\rangle\right. \\
+\left\langle D_{\gamma} \eta\left(\pi_{x^{\circ}}(t), \pi_{y}(t)\right), \frac{\partial V_{\alpha}}{\partial y_{\gamma}}\left(\pi_{y}(t), \lambda, \mu(t), v(t)\right)\right\rangle \\
\left.+\left\langle D_{\theta \sigma}^{2} \eta\left(\pi_{x^{\circ}}(t), \pi_{y}(t)\right), \frac{\partial V_{\alpha}}{\partial y_{\theta \sigma}}\left(\pi_{y}(t), \lambda, \mu(t), v(t)\right)\right\rangle\right] d t^{\alpha} \\
<-\left\|\theta\left(x^{\circ}(\cdot), y(\cdot)\right)\right\|^{2}\left(\rho^{\prime \ell} \Lambda_{\ell}^{10}+\rho^{\prime \prime \ell} \Lambda_{\ell}^{20}+\rho^{\prime \prime \prime}\right),
\end{aligned}
$$

where

$$
\begin{aligned}
V_{\alpha}\left(\pi_{y}(\cdot), \lambda, \mu(\cdot), v(\cdot)\right)= & \Lambda_{\ell}^{10} f_{\alpha}^{\ell}\left(\pi_{y}(t)\right)-\Lambda_{\ell}^{20} k_{\alpha}^{\ell}\left(\pi_{y}(t)\right) \\
& +\left\langle\mu_{\alpha}(y(t)), g\left(\pi_{y}(t)\right)\right\rangle+\left\langle v_{\alpha}(t), h\left(\pi_{y}(t)\right)\right\rangle, \quad t \in \Omega_{t_{0}, t_{1}}, \alpha=\overline{1, p} .
\end{aligned}
$$

The following relations hold:

$$
\begin{aligned}
& \left\langle D_{\gamma} \eta\left(\pi_{x^{\circ}}(t), \pi_{y}(t)\right), \frac{\partial V_{\alpha}}{\partial y_{\gamma}}\left(\pi_{y}(t), \lambda, \mu(t), v(t)\right)\right\rangle \\
& =D_{\gamma}\left\langle\eta\left(\pi_{x^{\circ}}(t), \pi_{y}(t)\right), \frac{\partial V_{\alpha}}{\partial y_{\gamma}}\left(\pi_{y}(t), \lambda, \mu(t), v(t)\right)\right\rangle \\
& \quad-\left\langle\eta\left(\pi_{x^{\circ}}(t), \pi_{y}(t)\right), D_{\gamma}\left(\frac{\partial V_{\alpha}}{\partial y_{\gamma}}\right)\left(\pi_{y}(t), \lambda, \mu(t), v(t)\right)\right\rangle,
\end{aligned}
$$




$$
\begin{aligned}
\left\langle D_{\theta \sigma}^{2}\right. & \left.\eta\left(\pi_{x^{\circ}}(t), \pi_{y}(t)\right), \frac{\partial V_{\alpha}}{\partial y_{\theta \sigma}}\left(\pi_{y}(t), \lambda, \mu(t), v(t)\right)\right\rangle \\
= & D_{\theta} D_{\sigma}\left\langle\eta\left(\pi_{x^{\circ}}(t), \pi_{y}(t)\right), \frac{\partial V_{\alpha}}{\partial y_{\theta \sigma}}\left(\pi_{y}(t), \lambda, \mu(t), v(t)\right)\right\rangle \\
& -D_{\theta}\left\langle\eta\left(\pi_{x^{\circ}}(t), \pi_{y}(t)\right), D_{\sigma} \frac{\partial V_{\alpha}}{\partial y_{\theta \sigma}}\left(\pi_{y}(t), \lambda, \mu(t), v(t)\right)\right\rangle \\
& -D_{\sigma}\left\langle\eta\left(\pi_{x^{\circ}}(t), \pi_{y}(t)\right), D_{\theta} \frac{\partial V_{\alpha}}{\partial y_{\theta \sigma}}\left(\pi_{y}(t), \lambda, \mu(t), v(t)\right)\right\rangle \\
& +\left\langle\eta\left(\pi_{x^{\circ}}(t), \pi_{y}(t)\right), D_{\sigma \theta}^{2} \frac{\partial V_{\alpha}}{\partial y_{\theta \sigma}}\left(\pi_{y}(t), \lambda, \mu(t), v(t)\right)\right\rangle .
\end{aligned}
$$

By replacing the relations (3.11) and (3.12), and using Euler-Lagrange PDE, the relation (3.9) becomes

$$
\begin{aligned}
\int_{\gamma_{t_{0}, t_{1}}}\left[D_{\gamma}\right. & \left\langle\eta\left(\pi_{x^{\circ}}(t), \pi_{y}(t)\right), \frac{\partial V_{\alpha}}{\partial y_{\gamma}}\left(\pi_{y}(t), \lambda, \mu(t), v(t)\right)\right\rangle \\
& +D_{\theta} D_{\sigma}\left\langle\eta\left(\pi_{x^{\circ}}(t), \pi_{y}(t)\right), \frac{\partial V_{\alpha}}{\partial y_{\theta \sigma}}\left(\pi_{y}(t), \lambda, \mu(t), v(t)\right)\right\rangle \\
& -D_{\theta}\left\langle\eta\left(\pi_{x^{\circ}}(t), \pi_{y}(t)\right), \frac{\partial V_{\alpha}}{\partial y_{\theta \sigma}}\left(\pi_{y}(t), \lambda, \mu(t), v(t)\right)\right\rangle \\
& \left.-D_{\sigma}\left\langle\eta\left(\pi_{x^{\circ}}(t), \pi_{y}(t)\right), D_{\theta} \frac{\partial V_{\alpha}}{\partial y_{\theta \sigma}}\left(\pi_{y}(t), \lambda, \mu(t), v(t)\right)\right\rangle\right] d t^{\alpha} \\
<- & \left\|\theta\left(x^{\circ}(\cdot), y(\cdot)\right)\right\|^{2}\left(\rho^{\prime \ell} \Lambda_{\ell}^{10}+\rho^{\prime \prime \ell} \Lambda_{\ell}^{20}+\rho^{\prime \prime \prime}\right) .
\end{aligned}
$$

According to [15], §9, a total divergence is equal to a total derivative, therefore, the left-hand side of (3.13) becomes null, and replacing into the inequality (3.13), it follows that

$$
0<-\left\|\theta\left(x^{\circ}(\cdot), y(\cdot)\right)\right\|^{2}\left(\rho^{\prime \ell} \Lambda_{\ell}^{10}+\rho^{\prime \prime \ell} \Lambda_{\ell}^{20}+\rho^{\prime \prime \prime}\right) .
$$

From hypothesis (e), the previous relation becomes $0<0$, which is false. Next, from relation (3.8), it follows that

$$
\begin{aligned}
0 \leqq & \Lambda_{\ell}^{10} F^{\ell}\left(x^{\circ}(\cdot)\right)-\Lambda_{\ell}^{20} K^{\ell}\left(x^{\circ}(\cdot)\right) \\
& +\int_{\gamma_{t_{0}, t_{1}}}\left[\left\langle\mu_{\alpha}(t), g\left(\pi_{x^{\circ}}(t)\right)\right\rangle+\left\langle v_{\alpha}(t), h\left(\pi_{x^{\circ}}(t)\right)\right\rangle\right] d t^{\alpha} \\
& -\int_{\gamma_{t_{0}, t_{1}}}\left[\left\langle\mu_{\alpha}(t), g\left(\pi_{y}(t)\right)\right\rangle+\left\langle v_{\alpha}(t), h\left(\pi_{y}(t)\right)\right\rangle\right] d t^{\alpha} .
\end{aligned}
$$


Taking into account the inequality:

$$
\int_{\gamma_{t_{0}, t_{1}}}\left[\left\langle\mu_{\alpha}(t), g\left(\pi_{y}(t)\right)\right\rangle+\left\langle v_{\alpha}(t), h\left(\pi_{y}(t)\right)\right\rangle\right] d t^{\alpha} \geqq 0,
$$

the above-mentioned relation becomes

$$
\Lambda_{\ell}^{10} F^{\ell}\left(x^{\circ}(\cdot)\right)-\Lambda_{\ell}^{20} K^{\ell}\left(x^{\circ}(\cdot)\right)>0,
$$

that is,

$$
K^{\ell}\left(x^{\circ}(\cdot)\right) K^{\ell}(y(\cdot))\left[\frac{F^{\ell}\left(x^{\circ}(\cdot)\right)}{K^{\ell}\left(x^{\circ}(\cdot)\right)}-\frac{F^{\ell}(y(\cdot))}{K^{\ell}(y(\cdot))}\right]>0 .
$$

Because $K^{\ell}\left(x^{\circ}(\cdot)\right) K^{\ell}(y(\cdot))>0, \ell=\overline{1, r}$, we conclude that

$$
\frac{F\left(x^{\circ}(\cdot)\right)}{K\left(x^{\circ}(\cdot)\right)}-\frac{F(y(\cdot))}{K(y(\cdot))} \not \leq(0, \ldots, 0),
$$

or

$$
\frac{F\left(x^{\circ}(\cdot)\right)}{K\left(x^{\circ}(\cdot)\right)} \not \leq \frac{F(y(\cdot))}{K(y(\cdot))} .
$$

Therefore, the relation $\varpi\left(x^{\circ}(\cdot)\right) \leq \delta\left(y(\cdot), y_{\gamma}(\cdot), \Lambda^{10}, \Lambda^{20}, \mu(\cdot), v(\cdot)\right)$ is not satisfied, and this completes the proof.

We will finish our considerations by giving the statements of two results on direct duality and converse duality, respectively. Their proofs follow directly from the weak duality.

Theorem 3.3 (Direct Duality). Let $x^{\circ}(\cdot)$ be an efficient solution of $(M F P)$. Suppose that the hypotheses of Theorem are satisfied. Then, there are $\Lambda^{10}$ and $\Lambda^{20}$ in $\mathbb{R}^{r}$, and the smooth functions $\mu^{\circ}$ : $\Omega_{t_{0}, t_{1}} \rightarrow \mathbb{R}^{m s p}$ and $v^{\circ}: \Omega_{t_{0}, t_{1}} \rightarrow \mathbb{R}^{q s p}$, such that $\left(x^{\circ}(\cdot), x_{\gamma}^{\circ}(\cdot), \Lambda^{10}, \Lambda^{20}, \mu^{\circ}(\cdot), v^{\circ}(\cdot)\right)$ is an efficient solution of the dual (MFD), and $\varpi\left(x^{\circ}(\cdot)\right)=\delta\left(x^{\circ}(\cdot), x_{\gamma}^{\circ}(\cdot), \Lambda^{10}, \Lambda^{20}, \mu^{\circ}(\cdot), v^{\circ}(\cdot)\right)$.

We will present now a theorem concerning the converse duality, by changing some of the hypotheses.

Theorem 3.4 (Converse Duality). Let $\left(x^{\circ}(\cdot), x_{\gamma}^{\circ}(\cdot), \Lambda^{10}, \Lambda^{20}, \mu^{\circ}(\cdot), \nu^{\circ}(\cdot)\right)$ be an efficient solution of the dual problem (MFD). Suppose the following conditions hold:

(a) $\bar{x}(\cdot)$ is an efficient solution of the primal problem $(M F P)$;

(b) for any $\ell=\overline{1, r}$, one has

$$
F^{\ell}\left(x^{\circ}(\cdot)\right)>0, \quad K^{\ell}\left(x^{\circ}(\cdot)\right)>0, \quad \Lambda_{\ell}^{10} F^{\ell}\left(x^{\circ}(\cdot)\right)-\Lambda_{\ell}^{20} K^{\ell}\left(x^{\circ}(\cdot)\right)=0 ;
$$


(c) for any $\ell=\overline{1, r}$, the functional $F^{\ell}(x(\cdot))$ is $\left(\rho^{\prime \ell}, b\right)$-quasiinvex at the point $x^{\circ}(\cdot)$ and the functional $-K^{\ell}(x(\cdot))$ is $\left(\rho^{\prime \prime \ell}, b\right)$-quasiinvex at the point $x^{\circ}(\cdot)$, with respect to $\eta$ and $\theta$;

(d) the functional,

$$
\int_{\gamma_{t_{0}, t_{1}}}\left[\left\langle\mu_{\alpha}(t), g\left(\pi_{x}(t)\right)\right\rangle+\left\langle v_{\alpha}(t), h\left(\pi_{x}(t)\right)\right\rangle\right] d t^{\alpha},
$$

is $\left(\rho^{\prime \prime \prime}, b\right)$-quasiinvex at the point $x^{\circ}(\cdot)$ with respect to $\eta$ and $\theta$;

(e) one of the functionals of $(c),(d)$ is strictly $\left(\rho^{\prime \ell}, b\right)-,\left(\rho^{\prime \prime \ell}, b\right)-$, or $\left(\rho^{\prime \prime \prime}, b\right)$-quasiinvex with respect to $\eta$ and $\theta$, respectively;

(f) $\rho^{\prime \ell} \Lambda_{\ell}^{10}+\rho^{\prime \prime \ell} \Lambda_{\ell}^{20}+\rho^{\prime \prime \prime} \geq 0$.

Then, $\bar{x}(\cdot)=x^{\circ}(\cdot)$, and $\varpi\left(x^{\circ}(\cdot)\right)=\delta\left(x^{\circ}(\cdot), x_{\gamma}^{\circ}(\cdot), \Lambda^{10}, \Lambda^{20}, \mu^{\circ}(\cdot), \nu^{\circ}(\cdot)\right)$.

\section{Conclusions}

In our previous work [12], we initiated an optimization theory for the second-order jet bundle. We considered the problem of minimization of vectors of curvilinear functionals (well known as mechanical work), thought as multitime multiobjective variational problem, subject to PDE and/or PDI constraints (limited resources). Within this framework, we introduced necessary conditions. As natural continuation of our results in [12], and strongly motivated by its possible applications in mechanics, the present work introduced a dual program theory for this class of problems (for related but complementary research, see [16, 17]).

\section{References}

[1] A. Chinchuluun and P. M. Pardalos, "A survey of recent developments in multiobjective optimization," Annals of Operations Research, vol. 154, pp. 29-50, 2007.

[2] G. R. Bitran, "Duality for nonlinear multiple-criteria optimization problems," Journal of Optimization Theory and Applications, vol. 35, no. 3, pp. 367-401, 1981.

[3] T. Tanino and Y. Sawaragi, "Duality theory in multiobjective programming," Journal of Optimization Theory and Applications, vol. 27, no. 4, pp. 509-529, 1979.

[4] R. R. Egudo, "Efficiency and generalized convex duality for multiobjective programs," Journal of Mathematical Analysis and Applications, vol. 138, no. 1, pp. 84-94, 1989.

[5] B. Mond and I. Husain, "Sufficient optimality criteria and duality for variational problems with generalised invexity," Journal of the Australian Mathematical Society B, vol. 31, no. 1, pp. 108-121, 1989.

[6] T. Weir and B. Mond, "Generalised convexity and duality in multiple objective programming," Bulletin of the Australian Mathematical Society, vol. 39, no. 2, pp. 287-299, 1989.

[7] B. Aghezzaf and M. Hachimi, "Generalized invexity and duality in multiobjective programming problems," Journal of Global Optimization, vol. 18, no. 1, pp. 91-101, 2000.

[8] V. Preda, "On efficiency and duality for multiobjective programs," Journal of Mathematical Analysis and Applications, vol. 166, no. 2, pp. 365-377, 1992.

[9] V. Preda, "On Mond-Weir duality for variational problems," Revue Roumaine de Mathématiques Pures et Appliquées, vol. 38, no. 2, pp. 155-164, 1993.

[10] R. I. Boț and S.-M. Grad, "Extending the classical vector Wolfe and Mond-Weir duality concepts via perturbations," Journal of Nonlinear and Convex Analysis, vol. 12, no. 1, pp. 81-101, 2011. 
[11] A. Pitea, C. Udrişte, and Ş. Mititelu, "PDI\&PDE-constrained optimization problems with curvilinear functional quotients as objective vectors," Balkan Journal of Geometry and Its Applications, vol. 14, no. 2, pp. 75-88, 2009.

[12] A. Pitea and M. Postolache, "Minimization of vectors of curvilinear functionals on the second order jet bundle. Necessary conditions," Optimization Letters. In press.

[13] C. Udrişte and M. Postolache, Atlas of Magnetic Geometric Dynamics, vol. 3, Geometry Balkan Press, Bucharest, Romania, 2001.

[14] C. Nahak and R. N. Mohapatra, "Nonsmooth $\rho-(\eta, \theta)$-invexity in multiobjective programming problems," Optimization Letters, vol. 6, no. 2, pp. 253-260, 2010.

[15] C. Udrişte, O. Dogaru, and I. Ţevy, "Null Lagrangian forms and Euler-Lagrange PDEs," Journal of Advanced Mathematical Studies, vol. 1, no. 1-2, pp. 143-156, 2008.

[16] A. Pitea and M. Postolache, "Minimization of vectors of curvilinear functionals on the second order jet bundle. Sufficient efficiency conditions," Optimization Letters. In press.

[17] A. Pitea and M. Postolache, "Duality theorems for a new class of multitime multiobjective variational problems," Journal of Global Optimization. In press. 


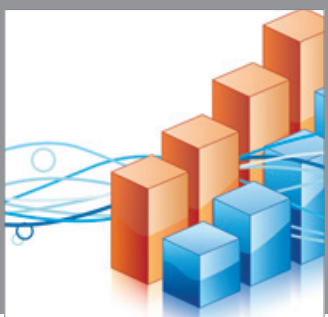

Advances in

Operations Research

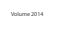

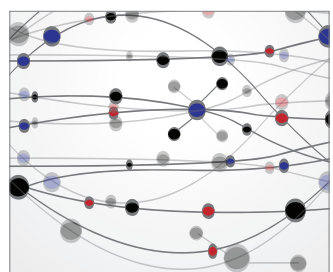

\section{The Scientific} World Journal
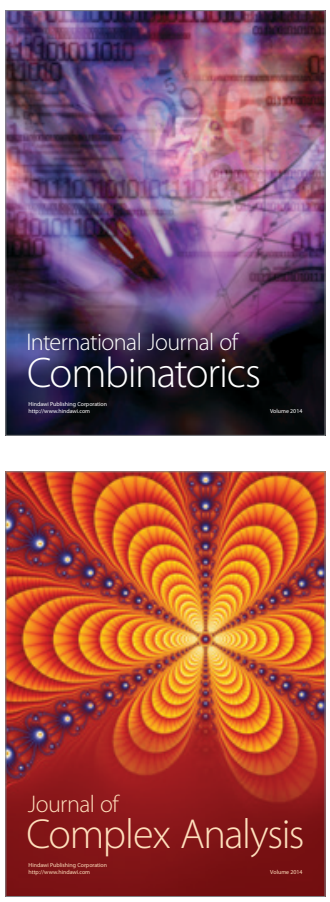

International Journal of

Mathematics and

Mathematical

Sciences
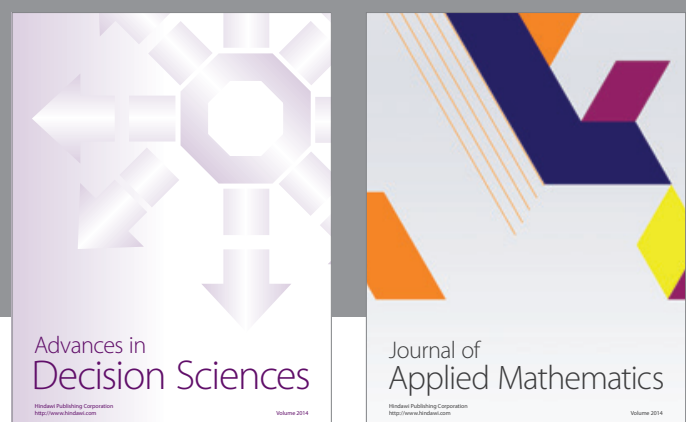

Journal of

Applied Mathematics
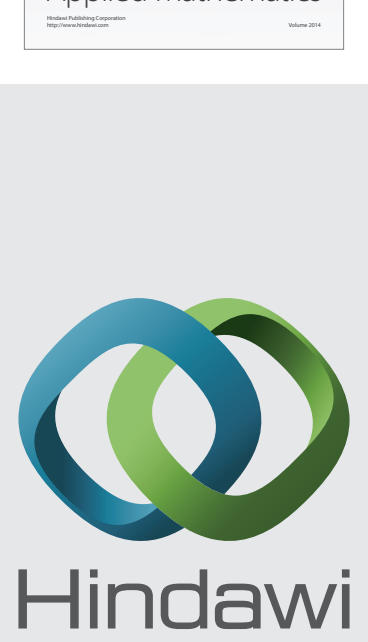

Submit your manuscripts at http://www.hindawi.com
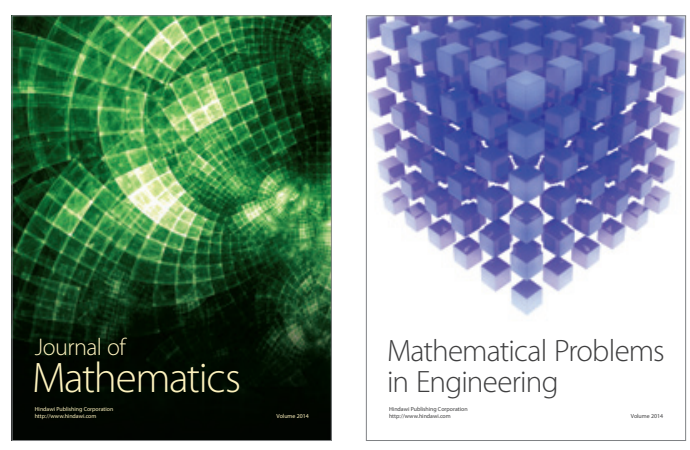

Mathematical Problems in Engineering
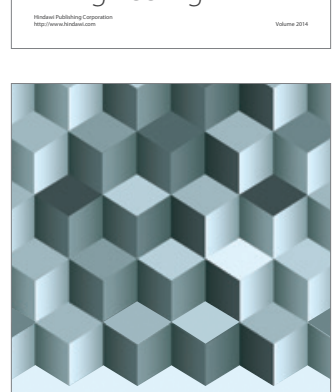

Journal of

Function Spaces
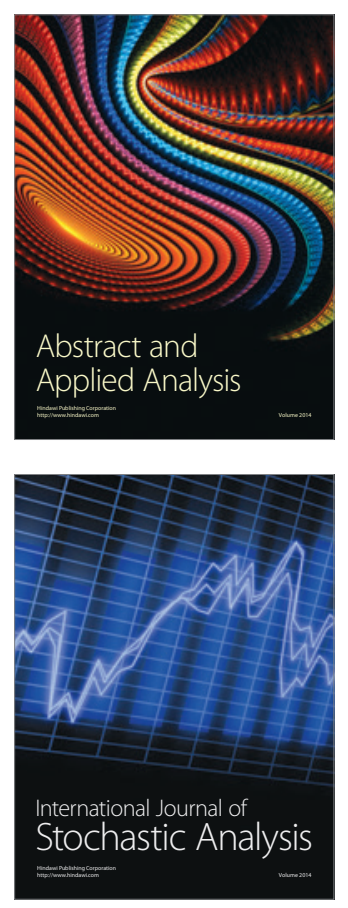

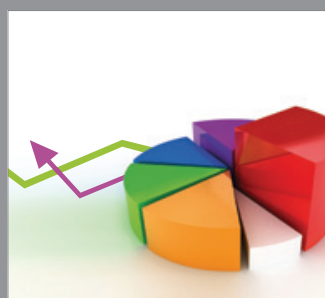

ournal of

Probability and Statistics

Promensencen
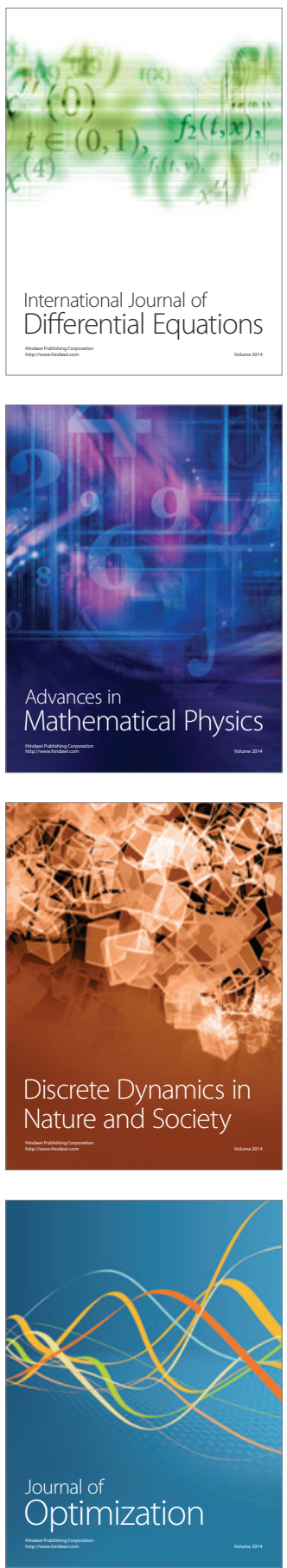